Document downloaded from:

http://hdl.handle.net/10251/179837

This paper must be cited as:

Nguyen, D.; Zvanovec, S.; Vallejo-Castro, L.; Ortega Tamarit, B.; Bohata, J.; Ghassemlooy, Z. (2020). Transmission of 2 bits/symbol over RoF and RoFSO links with different architectures for ubiquitous coverage. IEEE. 1-5. https://doi.org/10.1109/CSNDSP49049.2020.9249594

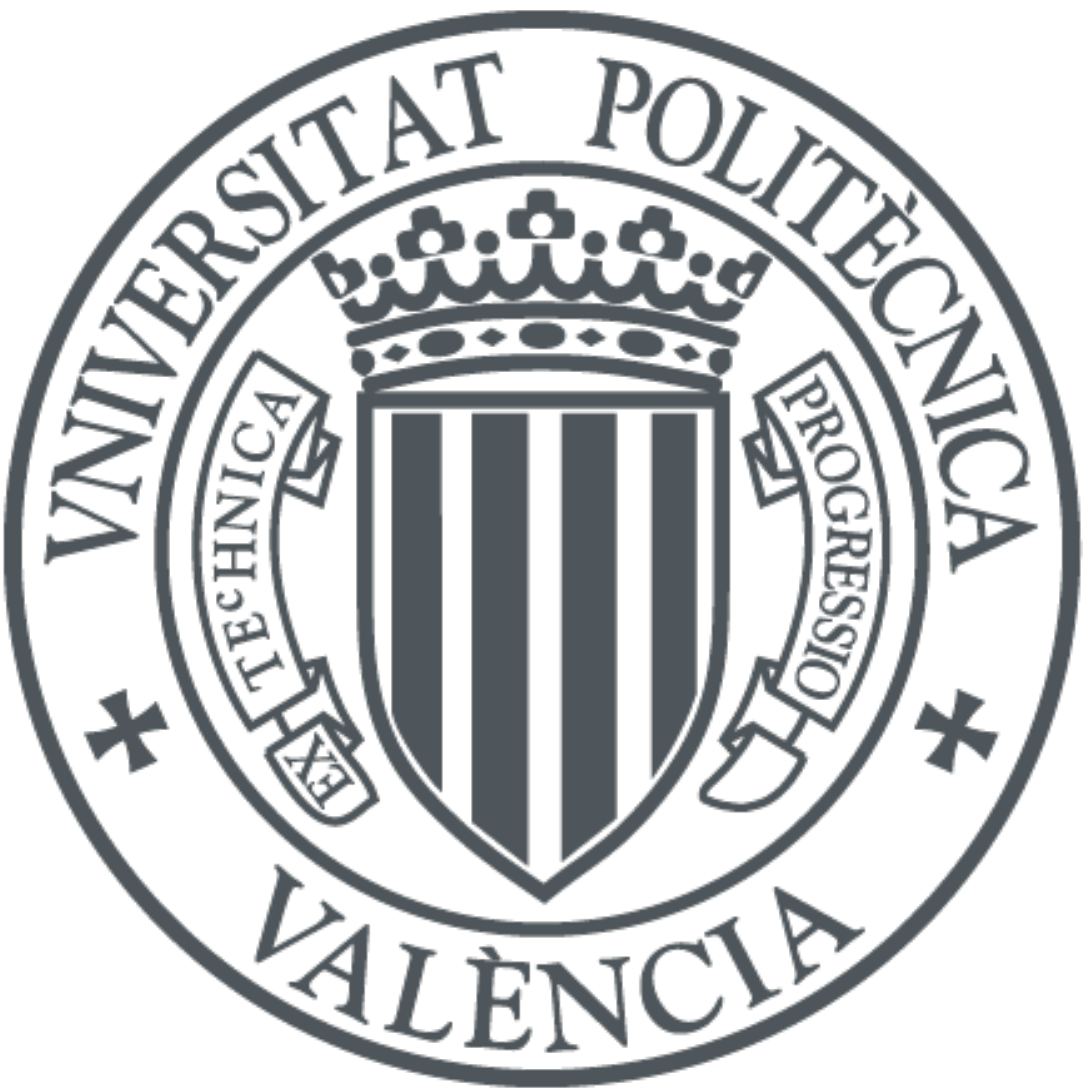

The final publication is available at

https://doi.org/10.1109/CSNDSP49049.2020.9249594

Copyright IEEE

Additional Information 


\section{Transmission of 2 bits/symbol over RoF and RoFSO links with different architectures for ubiquitous coverage}

\author{
Dong-Nhat Nguyen \\ Department of Electromagnetic Field \\ Czech Technical University in Prague \\ Prague, Czech Republic \\ dongnhat@fel.cvut.cz
}

Stanislav Zvanovec

Department of Electromagnetic Field

Czech Technical University in Prague

Prague, Czech Republic

xzvanove@fel.cvut.cz
Luis Vallejo

Instituto de Telecomunicaciones y

Aplicaciones Multimedia, ITEAM

Universitat Politècnica de València

Valencia, Spain

luivalc2@iteam.upv.es

Beatriz Ortega

Instituto de Telecomunicaciones y

Aplicaciones Multimedia, ITEAM

Universitat Politècnica de València

Valencia, Spain

bortega@dcom.upv.es

\author{
Jan Bohata \\ Department of Electromagnetic Field \\ Czech Technical University in Prague \\ Prague, Czech Republic \\ bohatja2@fel.cvut.cz \\ Zabih Ghassemlooy \\ Faculty of Engineering and \\ Environment \\ Northumbria University \\ Newcastle upon Tyne, United Kingdom \\ z.ghassemlooy@northumbria.ac.uk
}

\begin{abstract}
This paper investigates integrated radio-overfiber and radio-over-free-space optic links in a passive optical network architecture for ubiquitous wireless coverage. Two optical millimeter-wave generation techniques, namely optical heterodyning and optical modulator-based up-conversion are used. In the first configuration, simulation results are achieved for transmission of 20-40 Gb/s 4-PAM over the $60 \mathrm{GHz}$ heterodyned hybrid link. In the second configuration, transmission of $20 \mathrm{MHz}$ 4-QAM over the up-converted $25 \mathrm{GHz}$ hybrid system is examined. System performance evaluation and practical feasibility is carried out in terms of the received optical powers, bit error rates, eye diagrams, error vector magnitude and constellation diagrams.
\end{abstract}

Keywords—radio-over-fiber, free-space optics, passive optical networks, turbulence, millimeter-wave

\section{INTRODUCTION}

Current mobile networks deploy a large number of traditional tower-mounted macro base stations (BSs) where each BS covers a cell, processes and transmits its own signal. However, it is difficult to provide wide coverage to rural areas and underserved communities using these traditional BSs due to the high cost of installation and maintenance. Future mobile networks need to fulfil this demand apart from the higher data rates $R_{\mathrm{d}}$ and lower transmission latency. The cloud radio access network (C-RAN) with dense deployment of small-cell BSs (i.e., much smaller coverage over the traditional macro-cell BS) as a cost-effective architecture has attracted great interest from both the research community and the commercial sector over the past decade [1], [2]. C-RAN is considered as a typical realization of a mobile network supporting soft and green technologies in the future mobile networks since most of the signal processing, signal generation and modulation functions can be implemented at a central station (CS). This helps to simplify the antenna sites at the BSs with the remote radio head architecture. For that reason, C-RAN based on a radio-over-fiber (RoF) fronthaul link is highly applicable for small-cell and ultra-dense smallcell networks. Note that, RoF operating in the high radio frequency (RF) bands, such as in the millimeter wave ( $\mathrm{mmW}$ ) [3] and even terahertz-wave [4] have also recently gained tremendous attention for applications in $5^{\text {th }}$ generation $(5 \mathrm{G})$ mobile networks and beyond. The $\mathrm{mmW}$ signal can be optically generated by different techniques, although optical modulator-based up-conversion [5] and optical heterodyning [6] are the most well-known and widely used in the literature.

It is worth to mention that, the installation of optical fiber (OF) cables between the CS and BS within the RoF-based CRAN architecture can be challenging and costly. The use of OF cables is also impractical in many cases considering the geographical constraints and physical obstacles. The freespace optical (FSO) communication-based technology [7] could be therefore adopted to solve the aforementioned issues to maintain the seamless connectivity as a backup or a lastmile access link since the FSO technology offers unique features such as large bandwidth, license-free spectrum, high bit rate, easy and quick deployment. FSO transceivers operating at a wavelength of $1550 \mathrm{~nm}$, which is compatible with the $3^{\text {rd }}$ transmission window in $\mathrm{OF}$ communications (OFC), and $R_{\mathrm{d}}$ of up to $30 \mathrm{~Gb} / \mathrm{s}$ over a transmission distance $L$ of $1.5 \mathrm{~km}$ are commercially available [8]. Radio-over-FSO (RoFSO), therefore, has also been introduced and demonstrated since 2006 [9], where researchers from the University of Oklahoma, USA, transported the modulated analog RF signals within the range of 46 to $870 \mathrm{MHz}$ over an indoor $3 \mathrm{~m}$ FSO link and characterized the key performance parameters (i.e., carrier-to-noise ratio and spurious free dynamic range) without considering the effects of atmospheric phenomena such as turbulence. The turbulence strength is usually evaluated by the refractive index structure parameter $C_{n}^{2}\left(\mathrm{~m}^{-2 / 3}\right)$ [7]. $C_{n}^{2}$ can vary from $10^{-17}$ up to $10^{-12} \mathrm{~m}^{-2 / 3}$ for weak-to-strong turbulence regimes, respectively. It is well-known that FSO links are affected by 


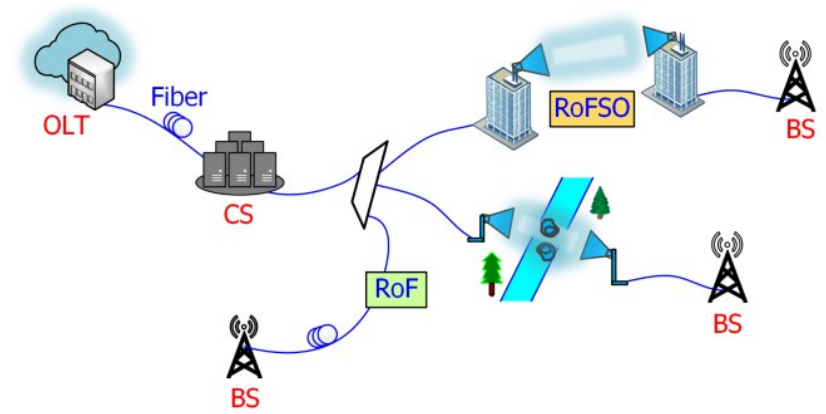

Figure 1. The simplified concept of delivering RF signal over hybrid RoF-FSO systems to remote areas in PON architecture.

turbulence strength, which may reduce the quality-of-service. Then, in 2009, researchers from Waseda University, Japan carried out a complete and long-term RoFSO measurements with RF ranging from $450 \mathrm{kHz}$ to $420 \mathrm{MHz}$ and a FSO link span of $1 \mathrm{~km}$ [10]. The link was demonstrated in an outdoor environment and the effect of turbulence was therefore comprehended. Seamless integration of RoF and RoFSO (i.e., RoF-FSO) operating in the $\mathrm{mmW}$ region are thus very attractive to deliver wide coverage in rural areas [11], [12]. We recently proposed and successfully demonstrated a $25 \mathrm{GHz}$ mmW link with 4/16/64-quadrature amplitude modulation (QAM) over an integrated RoF-FSO link with the FSO path being exposed to weak-to-strong turbulence regimes [13].

In this work, we propose and investigate the transmission of 2 bits/symbol using 4-level pulse amplitude modulation (4PAM) and 4-QAM over the seamless RoF-FSO in a passive optical network (PON) architecture to further scale the system mobility and reduce the cost for ubiquitous coverage, see Fig. 1. Note that, there are a number of benefits such as energy savings, scalability, signal security and higher split ratio that make $\mathrm{PON}$ the main and most extensively employed network in OFC systems. In addition, both 4-PAM and 4QAM have been evaluated as promising candidates for the next-generation PON [14], [15]. Regarding the optical mmW generation, two different schemes are considered at the CS in this work as follows:

1) Scheme A: Transmission of 4-PAM over a $60 \mathrm{GHz}$ hybrid link using optical heterodyning.

2) Scheme B: Transmisison of 4-QAM over a $25 \mathrm{GHz}$ hybrid link using optical Mach-Zehnder modulator (MZM)-based up-conversion.

Both frequencies are considered in $5 \mathrm{G}$ mobile networks [16]. Another goal of this work is also to evaluate the effect of both uniform and non-uniform turbulence on the FSO link and therefore the hybrid system performance.

The remaining part of the paper is structured as follows. Section II describes the simulation setup and results of Scheme A. Section III presents the experimental setup and results of Scheme B. Finally, the conclusion is given in Section IV.
II. Simulation SETUP AND Results Using 4-PAM With 60 GHZ MMW CARRIER FREQUENCY OF 60 GHz (SCHEME A)

\section{A. Simulation Setup}

Fig. 2 shows the simulation setup of 4-PAM transmission over the implemented system using the optical heterodyning technique for $\mathrm{mmW}$ signal generation. Note that, an experimental demonstration of this scheme has been carried out in [17], but only investigating transmission from OLT to $\mathrm{CS}$ to BS (ODN not included) and using low spectral efficiency non-return-to-zero signal.

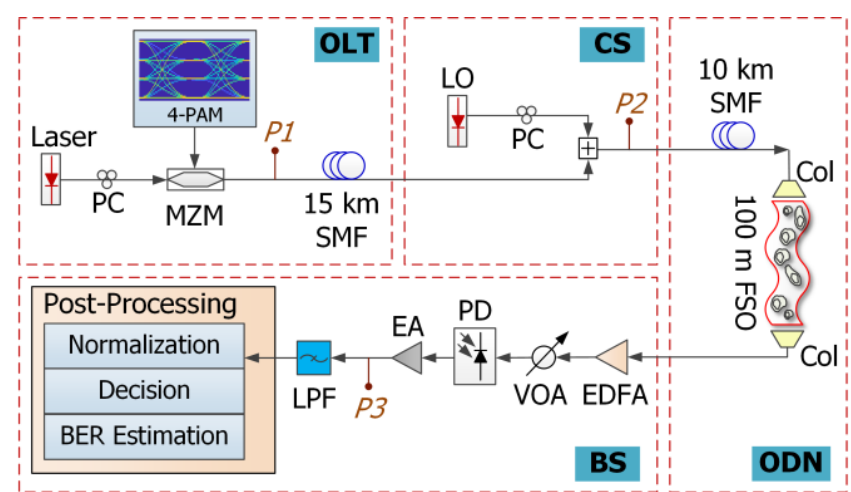

Figure 2. Simulation setup (Scheme A).

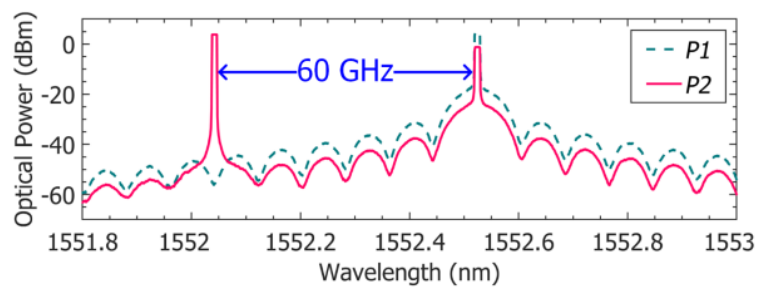

(a)

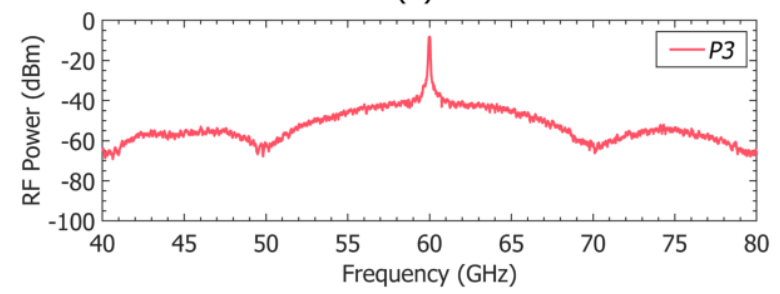

(b)

Figure 3. (a) Optical and (b) electrical spectra obtained at respective locations ( $P 1, P 2, P 3$ - see the setup in Fig. 2).

At the optical line terminal (OLT), an optical signal emitted by a distributed feedback (DFB) laser is applied through a polarization controller (PC) into a MZM, where it is externally modulated by an electrical 4-PAM signal at $20 \mathrm{~Gb} / \mathrm{s}$. The optical baseband signal is then transmitted over a $15 \mathrm{~km}$ standard single-mode fiber (SMF) representing a typical optical link. Optical heterodyning approach to generate an $\mathrm{mmW}$ signal at the $\mathrm{CS}$ is based on the combination of the optical baseband signal with another DFB laser (i.e., a local oscillator (LO) laser) which is set to a lasing frequency exactly $60 \mathrm{GHz}$ apart from the former one. The corresponding spectra at different positions in Scheme A are indicated in Fig. 3. The optical $\mathrm{mmW}$ signal is further transmitted over the optical distribution network (ODN), which consists of $10 \mathrm{~km} \mathrm{SMF}$ and $100 \mathrm{~m}$ FSO channel with weak uniform turbulence $C_{n}^{2}$ of $1.3 \times 10^{-16} \mathrm{~m}^{-2 / 3}$. Note that, the employed FSO collimators $(\mathrm{Col})$ parameters are 
based on commercial devices [8]. The optical signal is then amplified by using an erbium-doped fiber amplifier (EDFA) and then directly detected by a PIN photodiode (PD), which has a responsivity of $0.6 \mathrm{~A} / \mathrm{W}$ and a dark current of $10 \mathrm{nA}$, for opto-electronic conversion at the BS. A variable optical attenuator (VOA) is used to adjust the received optical power $P_{r}$ at the the PD. The recovered electrical signal is then amplified by a low-noise electrical amplifier (EA), which has a $30 \mathrm{~dB}$ gain, followed by a low-pass Bessel filter (LPF). Finally, the signal is post-processed in MATLAB-based digital signal processing module to carry out normalization, symbol decision and bit error rate (BER) estimation. The main parameters adopted in the simulation are summarized in Table 1.

Table 1. Main simulation parameters (Scheme A).

\begin{tabular}{|l|l|}
\hline Parameter & Value \\
\hline $\begin{array}{l}\text { Laser } 1 \\
\text { - Wavelength } \\
\text { - Output power } \\
\text { Laser 2 (LO) } \\
\text { - Wavelength } \\
\text { - Output power }\end{array}$ & $1552.524 \mathrm{~nm}$ \\
\hline Modulation format & $16 \mathrm{dBm}$ \\
\hline mmW carrier frequency & $1552.042 \mathrm{~nm}$ \\
\hline $\begin{array}{l}\text { SMF } \\
\text { - Length in OLT }\end{array}$ & $6.8 \mathrm{dBm}$ \\
- Length in ODN & $60 \mathrm{GHz}$ \\
- Dispersion & $15 \mathrm{~km}$ \\
\hline $\begin{array}{l}\text { FSO } \\
\text { - Aperture diameter }\end{array}$ & $10 \mathrm{~km}$ \\
- Length & $17 \mathrm{ps} /(\mathrm{nm} \cdot \mathrm{km})$ \\
\hline EDFA & $10 \mathrm{~cm}$ \\
- Output power & $100 \mathrm{~m}$ \\
\hline N Noise figure & $1 \mathrm{dBm}$ \\
\hline
\end{tabular}

\section{B. Simulation Results}

To investigate the maximal allowable transmission capacity of the hybrid link (Scheme A) when delivering 4-PAM, we show the BER versus the $R_{\mathrm{d}}$ of the received 4-PAM signal over the hybrid transmission link at $P_{\mathrm{r}}$ of $1 \mathrm{dBm}-$ see Fig. 4. As expected, the BER increases with the $R_{\mathrm{d}}$, where a maximum $R_{\mathrm{d}}$ of about $30 \mathrm{~Gb} / \mathrm{s}$ can be achieved at the forward error correction (FEC) BER limit of $3.8 \times 10^{-3}$.

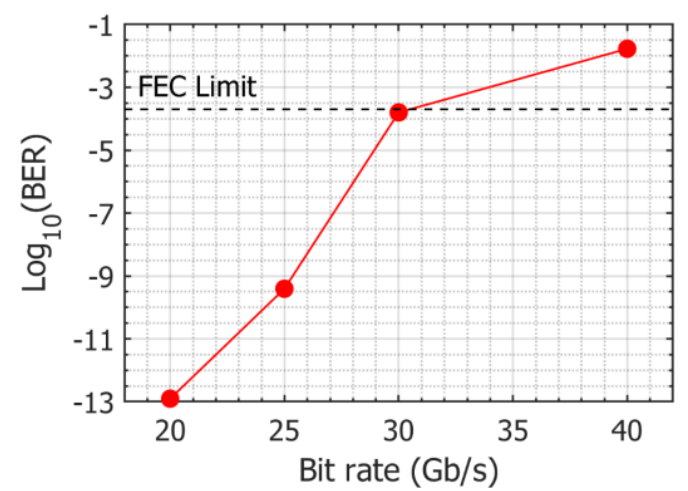

Figure 4. BER of 4-PAM signal after hybrid transmision at different bit rates obtained at $P_{\mathrm{r}}$ of $1 \mathrm{dBm}$ and $C_{n}^{2}$ of $1.3 \times 10^{-16} \mathrm{~m}^{-2 / 3}$.
A set of the corresponding received 4-PAM eye diagrams at $R_{\mathrm{d}}$ of $20,25,30$ and $40 \mathrm{~Gb} / \mathrm{s}$ obtained at $P_{\mathrm{r}}$ of $1 \mathrm{dBm}$ is shown in Figs. 5 (a)-(d), respectively. As $R_{\mathrm{d}}$ increases, the eye diagrams accumulate more intersymbol interference, which has an impact on the corresponding BER as depicted in Fig. 4.

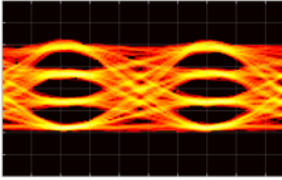

(a)

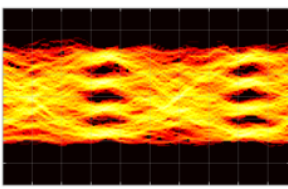

(c)

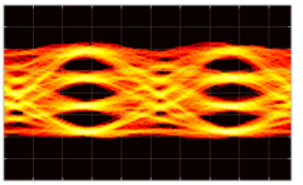

(b)

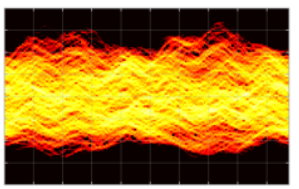

(d)
Figure 5. Eye diagrams of 4-PAM signal after transmission captured at $P_{\mathrm{r}}$ of $1 \mathrm{dBm}$ with a data rate of (a) $20 \mathrm{~Gb} / \mathrm{s}$, (b) $25 \mathrm{~Gb} / \mathrm{s}$, (c) $30 \mathrm{~Gb} / \mathrm{s}$ and (d) $40 \mathrm{~Gb} / \mathrm{s}$.

To further study the performance of $20 \mathrm{~Gb} / \mathrm{s} 4-\mathrm{PAM}$ under the effect of turbulence over a $100 \mathrm{~m}$ FSO channel for the proposed hybrid link, we consider both uniform and nonuniform turbulence scenarios as can be seen in Fig. 6. In detail, for the former, we set $C_{n}^{2}$ of $2.0 \times 10^{-15} \mathrm{~m}^{-2 / 3}$ along a $100 \mathrm{~m}$ FSO link to mimic the moderate uniform turbulence. For the latter, we have considered a strong turbulence (i.e., $C_{n}^{2}$ of $1.6 \times 10^{-13} \mathrm{~m}^{-2 / 3}$ ) at the center of the FSO link (i.e., $40 \mathrm{~m}$ ) and weak turbulence (i.e., $C_{n}^{2}$ of $1.3 \times 10^{-16} \mathrm{~m}^{-2 / 3}$ ) for both ends of the channel in order to create a non-uniform turbulence which the link may experience in real practical outdoor environments.

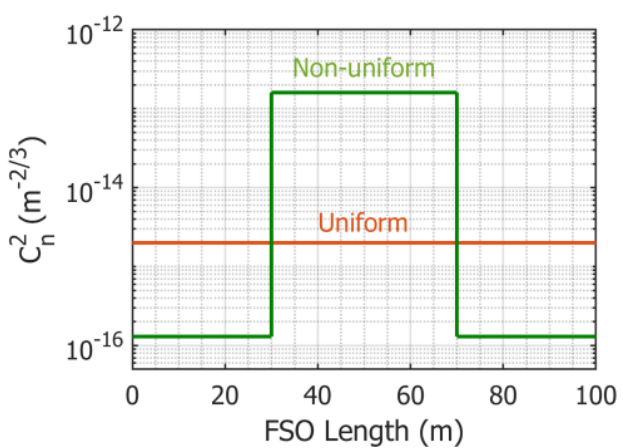

Figure 6. Turbulence scenarios considered in the simulation.

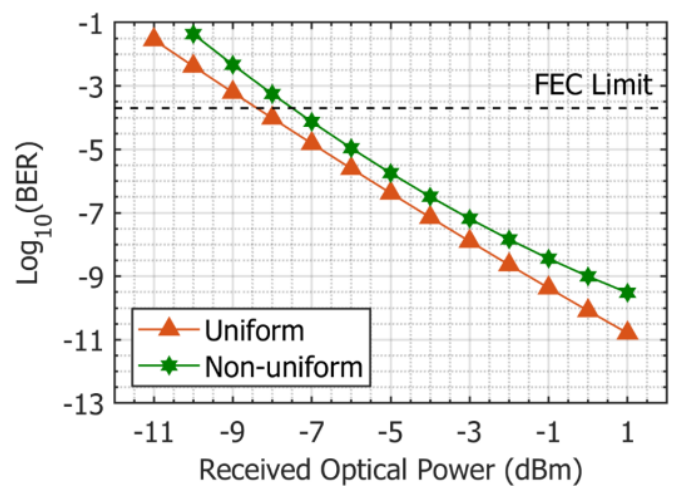

Figure 7. BER versus $P_{\mathrm{r}}$ for 4-PAM transmisison at $20 \mathrm{~Gb} / \mathrm{s}$ with different turbulence scenarios. 
Fig. 7 compares the simulated BERs as a function of $P_{\mathrm{r}}$ for the two considered turbulence scenarios. The BER is, in both cases, well below the FEC limit for $P_{\mathrm{r}}$ larger than -7 $\mathrm{dBm}$. As can be clearly seen, at the FEC BER limit there is $1 \mathrm{~dB}$ power penaty for the link with non-uniform turbulence compared with the channel with uniform turbulence. Compared to weak uniform turbulence $-C_{n}^{2}$ of $1.3 \times$ $10^{-16} \mathrm{~m}^{-2 / 3}$ as shown in Fig. 4, moderate uniform turbulence with $C_{n}^{2}$ of $2 \times 10^{-15} \mathrm{~m}^{-2 / 3}$ and non-uniform turbulence result in more than two and three orders of magnitude degradation in BER.

\section{EXPERIMENTAL SETUP AND RESULTS USING 4-QAM SIGNAL WITH MMW CARRIER FREQUENCY OF $25 \mathrm{GHz}$ (SCHEME B)}

\section{A. Experimental Setup}

The experimental setup of a hybrid RoF-FSO link using 4QAM with optical MZM-based up-conversion technique is shown in Fig. 8. At the OLT, the optical signal from the laser (ID Photonics CoBrite-DX4) is externally modulated by 4QAM with an intermediate frequency of $200 \mathrm{MHz}$, generated by a vector signal generator (R\&S SMW 200A) and then transmitted over a $15 \mathrm{~km}$ of SMF. Unlike Scheme A, which uses optical heterodyning at the CS, in the Scheme B, another MZM (MZM 2) is used for up-conversion. MZM 2 is externally modulated by $12.5 \mathrm{GHz}$ RF clock from a signal generator (R\&S SMF 100A). Note that, it is biased at the minimum transmission point, and therefore the optical spectrum with suppressed carrier at the output of MZM 2 and the frequency spacing of $25 \mathrm{GHz}$ between two sidebands are achieved, see Fig. 9. The carrier suppression ratio of about $23 \mathrm{~dB}$ is obtained. The optically amplified signal is then transmitted over ODN, which consists of $10 \mathrm{~km} \mathrm{SMF}$ and $2 \mathrm{~m}$ FSO channel within an indoor atmospheric chamber.

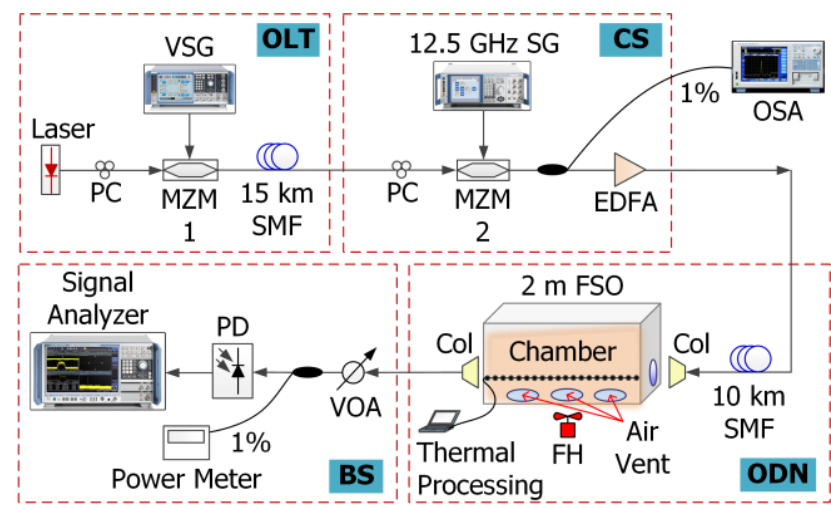

Figure 8. Experimental setup (Scheme B).

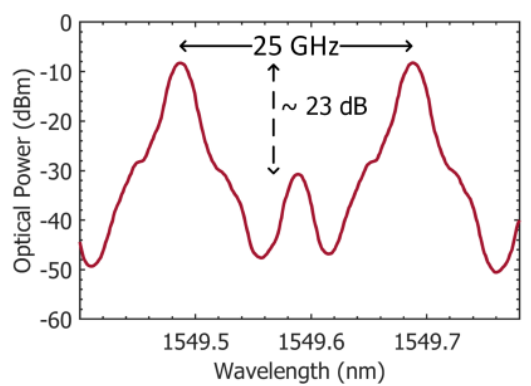

Figure 9. Optical spectrum featuring double-sideband with optical carrier suppression measured at the MZM 2 output.
Non-uniform turbulence is generated using a fan heater (FH), which blows hot air to the middle of chamber via the central air vent. To accurately measure the thermal profiles and then determine $C_{n}^{2}, 20$ thermal sensors are used, see measurements in Fig. 10. More detail on $C_{n}^{2}$ measurements within the chamber can be found in [13]. At the BS, the optical signal is detected using a high-speed PD, the output of which evaluated using a signal analyzer. The key experimental parameters are summarized in Table 2.

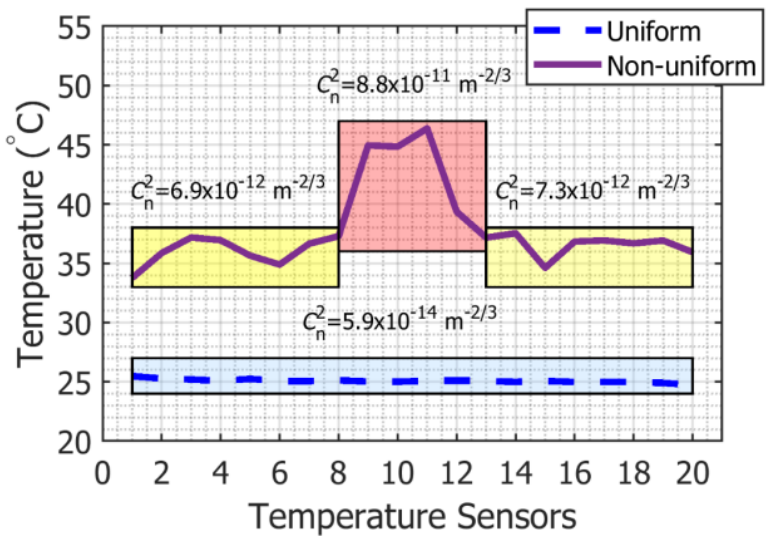

Figure 10. Turbulence scenarios considered in the experiment.

Table 2. Main experimental parameters (Scheme B).

\begin{tabular}{|l|l|}
\hline Parameter & Value \\
\hline $\begin{array}{l}\text { Laser } \\
\text { - Wavelength }\end{array}$ & $1549.6 \mathrm{~nm}$ \\
- Output power & $16 \mathrm{dBm}$ \\
\hline Modulation format & $4-\mathrm{QAM}$ \\
\hline mmW carrier frequency & $25 \mathrm{GHz}$ \\
\hline SMF & \\
- Length in OLT & $15 \mathrm{~km}$ \\
- Length in ODN & $10 \mathrm{~km}$ \\
- Dispersion & $17 \mathrm{ps} /(\mathrm{nm} \cdot \mathrm{km})$ \\
\hline $\begin{array}{l}\text { FSO } \\
\text { - Aperture diameter }\end{array}$ & $2.54 \mathrm{~cm}$ \\
- Length & $2 \mathrm{~m}$ \\
\hline EDFA & \\
- Output power & $6 \mathrm{dBm}$ \\
- Noise figure & $<4 \mathrm{~dB}$ \\
\hline
\end{tabular}

\section{B. Experimental Results}

Fig. 11 shows the measured error vector magnitude (EVM) as a function of $P_{\mathrm{r}}$ for $20 \mathrm{MHz}$ 4-QAM following transmission over the hybrid link (Scheme B) for different turbulence scenarios. Also shown is the $18.5 \%$ EVM required limit for 4QAM according to the third-generation partnership project (3GPP) specifications [18].

Apparently, at $P_{\mathrm{r}}$ of $6 \mathrm{dBm}$, the EVMs of 4-QAM under uniform and non-uniform turbulences are 9.5 and $13 \%$, respectively, which leads to an EVM degradation of 3.5\%. Both EVM values, however, are well below EVM requirements. The results also indicate less than $1 \mathrm{~dB}$ optical power degradation at the EVM limit of $18.5 \%$ due to the influence of non-uniform turbulence in the FSO channel. 


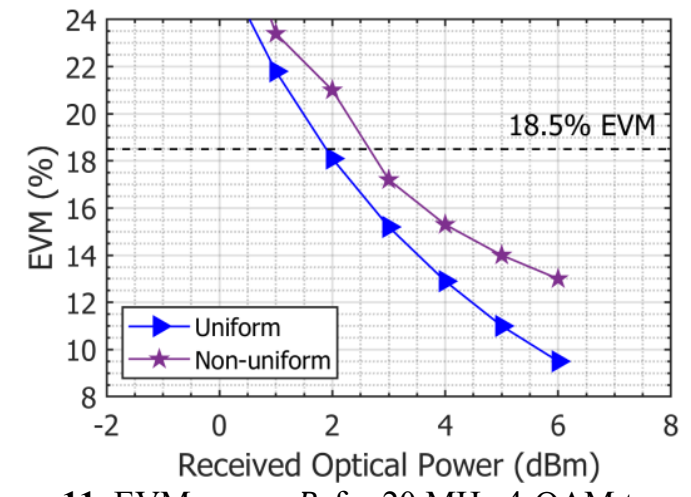

Figure 11. EVM versus $P_{\mathrm{r}}$ for $20 \mathrm{MHz} 4-\mathrm{QAM}$ transmisison with different $C_{n}^{2}$.

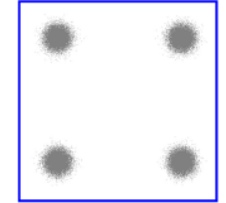

(a)

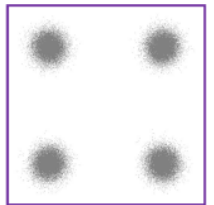

(b)
Figure 12. Constellation diagrams captured at $P_{\mathrm{r}}$ of $6 \mathrm{dBm}$ of $20 \mathrm{MHz} 4-\mathrm{Q} A M$ signal after tranmission under (a) uniform and (b) non-uniform turbulence, respectively.

To further demonstrate the effect of uniform and nonuniform turbulence on the signal quality, we show in Fig. 12 the measured constellation diagrams of the received 4-QAM measured at $P_{\mathrm{r}}$ of $6 \mathrm{dBm}$. Under non-uniform turbulence, Fig. 12(b), the 4-QAM symbol size is more diffused in comparison to the constellation under uniform turbulence shown in Fig. 12(a). This corresponds to simulation results as presented in Section II.

\section{CONCLUSIONS}

We analyzed two different hybrid RoF-FSO transmission links based on optical heterodyning and optical modulatorbased up-conversion techniques for ubiquitous broadband wireless access. Two bits/symbol signals namely 4-PAM and 4-QAM were transmitted over the proposed systems. The impact of FSO channel under the effect of turbulence is also examined in detail. At the maximum $P_{\mathrm{r}}$, for the simulation results with $20 \mathrm{~Gb} / \mathrm{s}$ 4-PAM, the effect of non-uniform turbulence caused more than one order of magnitude degradation in BER compared to uniform turbulence. While for $20 \mathrm{MHz}$ 4-QAM transmission in experiment, the EVM penalty beween non- and uniform turbulence is about 3.5\%. Both experimental and simulation results therefore showed good agreement regarding the impact of non-uniform turbulence, which is more severe in comparison to the uniform turbulence on the system performance.

\section{ACKNOWLEDGMENT}

This work is supported by International Mobility of Researchers in CTU (CZ.02.2.69/0.0/0.0/16 027/0008465) and MEYS INTER-COST project (within LTC 18008 ).

\section{REFERENCES}

[1] China Mobile Reserach Institute, "C-RAN: the road towards green RAN, white paper," 2011.

[2] I. A. Alimi, A. L. Teixeira, and P. P. Monteiro,
"Toward an efficient C-RAN optical fronthaul for the future networks: A tutorial on technologies, requirements, challenges, and solutions," IEEE Commun. Surv. Tutorials, vol. 20, no. 1, pp. 708769, 2018.

[3] P. T. Dat, A. Kanno, and N. Yamamoto, "Seamless convergence of fiber and wireless systems for $5 \mathrm{G}$ and beyond networks," J. Lightwave Technol., vol. 37, no. 2, pp. 592-605, 2019.

[4] T. Kawanishi, "THz and photonic seamless communications," J. Lightwave Technol., vol. 37, no. 7, pp. 1671-1679, 2019.

[5] L. Vallejo et al., "Impact of thermal-induced turbulent distribution along FSO link on transmission of photonically generated $\mathrm{mmW}$ signals in the frequency range 26-40 GHz," IEEE Photonics J., vol. 12, no. 1, pp. 1-9, Feb. 2020.

[6] E. Martin et al., " $28 \mathrm{GHz} 5 \mathrm{G}$ radio over fibre using UF-OFDM with optical heterodyning," in International Topical Meeting on Microwave Photonics (MWP), 2017, p. Th2.4.

[7] Z. Ghassemlooy, W. O. Popoola, and S. Rajbhandari, Optical wireless communications - system and channel modelling with Matlab, 2nd ed. Cham: CRC Press, 2019.

[8] "30 Gbit/s ARTOLINK." [Online]. Available: http://artolink.com/.

[9] H. H. Refai, J. J. Sluss, Jr., and H. H. Refai, "Comparative study of the performance of analog fiber optic links versus free-space optical links," Opt. Eng., vol. 45, no. 2, p. 025003, Feb. 2006.

[10] P. T. Dat et al., "Studies on characterizing the transmission of RF signals over a turbulent FSO link," Opt. Express, vol. 17, no. 10, p. 7731, 2009.

[11] P. T. Dat, A. Kanno, K. Inagaki, T. Umezawa, N. Yamamoto, and T. Kawanishi, "Hybrid optical wireless-mmWave: ultra high-speed indoor communications for beyond 5G," in IEEE INFOCOM 2019 - IEEE Conference on Computer Communications Workshops (INFOCOM WKSHPS), 2019, pp. 1003-1004.

[12] Y. Alfadhli et al., "Real-time FPGA demonstration of hybrid bi-directional MMW and FSO fronthaul architecture," in 2019 Optical Fiber Communications Conference and Exhibition, OFC 2019 Proceedings, 2019, p. W2A.39.

[13] D.-N. Nguyen, J. Bohata, M. Komanec, S. Zvanovec, B. Ortega, and Z. Ghassemlooy, "Seamless $25 \mathrm{GHz}$ transmission of LTE 4/16/64-QAM signals over hybrid SMF/FSO and wireless link," J. Lightwave Technol., vol. 37, no. 24, pp. 6040-6047, 2019.

[14] R. Van Der Linden, N. C. Tran, E. Tangdiongga, and T. Koonen, "Optimization of flexible non-uniform multilevel PAM for maximizing the aggregated capacity in PON deployments," J. Lightwave Technol., vol. 36, no. 12, pp. 2328-2336, 2018.

[15] K. Matsuda and N. Suzuki, "Hardware-efficient signal processing technologies for coherent PON systems," J. Lightwave Technol., vol. 37, no. 6, pp. 1614-1620, 2019.

[16] “5G spectrum GSMA public policy position," 2018. [Online]. Available: https://www.gsma.com/. 
[17] S. Rommel et al., "Outdoor W-Band hybrid photonic wireless link based on an optical SFP + module," IEEE Photonics Technol. Lett., vol. 28, no. 21, pp. 2303-2306, 2016.

[18] "Universal Mobile Telecommunications System
(UMTS); LTE; Active Antenna System (AAS) Base Station (BS) conformance testing; Part 1: conducted conformance testing (3GPP TS 37.145-1 version 14.1.0 Release 14)," 2017. 